\title{
Impact of corticosteroid therapy on outcomes of persons with SARS-CoV-2, SARS-CoV, or MERS-CoV infection: a systematic review and meta-analysis
}

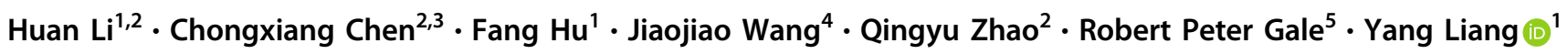

Received: 7 April 2020 / Revised: 22 April 2020 / Accepted: 22 April 2020 / Published online: 5 May 2020

(c) The Author(s), under exclusive licence to Springer Nature Limited 2020

\begin{abstract}
We performed a meta-analysis to determine safety and efficacy of corticosteroids in SARS-CoV-2, SARS-CoV, and MERS-CoV infections. We searched PubMed, Web of Science, Medline, WanFang Chinese database, and ZhiWang Chinese database using Boolean operators and search terms covering SARS-CoV-2, SARS-CoV, OR MERS-CoV AND corticosteroids to find appropriate studies. Review Manager 5.3 was used to analyze results of meta-analysis. Observational studies were analyzed for quality using the modified Newcastle-Ottawa scale and randomized clinical trials, using the Jadad scale. Subjects were divided into those with severe-only and other (severe and not severe) cohorts based on published criteria. Efficacy endpoints studied included mortality, hospitalization duration, rates of intensive care unit (ICU) admission, use of mechanical ventilation, and a composite endpoint (death, ICU admission, or mechanical ventilation). We included 11 reports including 10 cohort studies and 1 randomized clinical trial involving 5249 subjects (2003-2020). Two discussed the association of corticosteroids and virus clearing and 10 explored how corticosteroids impacted mortality, hospitalization duration, use of mechanical ventilation, and a composite endpoint. Corticosteroid use was associated with delayed virus clearing with a mean difference (MD) $=3.78$ days (95\% confidence Interval $[\mathrm{CI}]=1.16,6.41$ days; $\left.I^{2}=0 \%\right)$. There was no significant reduction in deaths with relative Risk Ratio $(\mathrm{RR})=$ $1.07\left(90 \% \mathrm{CI}=0.81 ; 1.42 ; I^{2}=80 \%\right)$. Hospitalization duration was prolonged and use of mechanical ventilation increased. In conclusion, corticosteroid use in subjects with SARS-CoV-2, SARS-CoV, and MERS-CoV infections delayed virus clearing and did not convincingly improve survival, reduce hospitalization duration or ICU admission rate and/or use of mechanical ventilation. There were several adverse effects. Because of a preponderance of observational studies in the dataset and selection and publication biases our conclusions, especially regarding SARS$\mathrm{CoV}-2$, need confirmation in a randomized clinical trial. In the interim we suggest caution using corticosteroids in persons with COVID-19.
\end{abstract}

These authors contributed equally: Huan Li, Chongxiang Chen

Supplementary information The online version of this article (https:// doi.org/10.1038/s41375-020-0848-3) contains supplementary material, which is available to authorized users.

Robert Peter Gale

robertpetergale@alumni.ucla.edu

$\triangle$ Yang Liang

liangyang@sysucc.org.cn

1 Department of Hematologic Oncology, State Key Laboratory of Oncology in South China; Collaborative Innovation Center for Cancer Medicine, Sun Yat-sen University Cancer Center, Guangzhou, 510060 Guangdong Province, China

2 Department of ICU, State Key Laboratory of Oncology in South China; Collaborative Innovation Center for Cancer Medicine, Sun

\section{Introduction}

In December 2019, Wuhan, Hubei province, China, became the epicenter of a pandemic caused by severe

Yat-sen University Cancer Center, Guangzhou, 510060

Guangdong Province, China

3 State Key Laboratory of Respiratory Disease, Guangzhou Institute of Respiratory Health, First Affiliated Hospital of Guangzhou Medical University, Guangzhou, 510120 Guangdong Province, China

4 Department of Tuberculosis, Fuzhou Pulmonary Hospital, Teaching Hospital of Fujian Medical University, Fuzhou, 350008 Fujian Province, China

5 Department of Immunology and Inflammation, Haematology Research Centre, Imperial College London, London, UK 
acute respiratory syndrome coronavirus-2 (SARS-CoV-2) [1] which causes coronavirus infectious disease-2019 (COVID-19). About 15\% of cases of COVID-19 become severe [2]. About one-half of hospitalized persons with COVID-19 treated in the intensive care unit (ICU) receive corticosteroids versus $10-20 \%$ of hospitalized, non-ICU persons $[3,4]$.

There are several reports of using corticosteroids in the setting of severe coronavirus infections including SARS-CoV2, severe acute respiratory syndrome (SARS) and Middle East respiratory syndrome (MERS). There are few data on safety and efficacy of corticosteroids in COVID-19 and its use in SARS and MERS infections is controversial [5, 6].

SARS-CoV-2, SARS-CoV, and MERS-CoV shares many genetic features and SARS-CoV-2 is highly homologous to SARS-CoV [7]. Reports of efficacy of corticosteroid in some persons with SARS-CoV infections have resulted in widespread use of this therapy to treat COVID-19, especially for persons with severe infection hospitalized in the ICU. Presently, there are few data on safety and efficacy of corticosteroids in this setting [2, 810]. Because of the overlapping genetic and clinical feature of SARS-CoV-2, SARS-CoV, and MERS-CoV, we performed a meta-analysis of safety and efficacy of corticosteroid use in these coronavirus infections.

\section{Methods}

Our meta-analysis focus on the effects of corticosteroids on virus clearing and mortality in persons infected with SARS-CoV-2, SARS-CoV, or MERS-CoV. We searched PubMed, Web of Science, Medline, Chinese database WanFang, and ZhiWang using the terms: "coronavirus," OR “COVID-19," OR “2019-nCoV," OR "SARS-CoV-2" OR "severe acute respiratory syndrome," OR "Middle East respiratory syndrome," OR "SARS," OR "MERS" AND "corticosteroid" OR "glucocorticoid," "hydrocortisone" OR "methylprednisolone" OR "dexamethasone" OR "steroid" with these Boolean operators. Searches were done on March 20, 2020. Two investigators independently reviewed the identified abstracts and selected articles for full review. Discordances were resolved by a third investigator.

We identified 8788 publications. We next excluded 2057 duplicate reports. The remaining 6731 publications were scanned by 3 investigators who identified 159 articles relevant publications after considering title and abstract. In total, 11 publications met our selection criteria, 10 cohort studies [2, 8-16] and 1 randomized clinical trial [6]. We excluded studies from same geographical region to avoid duplication. Because most studies were observational the possibility of duplicate reporting was addressed by deleting small studies from the same geographical region. Publication flow and reasons for exclusions are displayed in Fig. 1 using PRIZMA flow diagram.

\section{Inclusion and exclusion criteria}

Inclusion criteria included: (1) research articles including observational studies and clinical trials but excluding reviews or case reports on the use of corticosteroids in persons with SARS-CoV-2, SARS-CoV, and MERS-CoV infections; (2) reported outcomes of virus clearance and/or death; and (3) published in English and/or Chinese. Studies with insufficient data were excluded.

\section{Covariates studied}

For each selected publication the following were extracted: (1) 1st author; (2) publication year; (3) country; (4) sample size; (5) subject-related covariates; (6) dose and duration of corticosteroid treatment; and (7) dose of corticosteroids. These data are displayed in Table 1. Primary outcome measures were rate of virus clearing and death. Secondary outcomes were duration of hospitalization, use of mechanical ventilation, composite endpoint, and corticosteroid use between severe-only and nonsevere subjects. The study used anonymized, published data requiring no Ethics Committee approval.

\section{Risk of bias}

The methodological quality of retrospective studies was assessed by the modified Newcastle-Ottawa scale (NOS) [17, 18] which consists of three domains: (1) subject selection; (2) comparability of the study groups; and (3) assessment of outcome(s). A score of 0-9 was allocated to each study except the randomized clinical trial. We considered observational studies with an NOS score $\geq 6$ high quality. We included 1 study with an NOS score of 5 because of a large sample size ( $>1000$ subjects) but performed sensitivity analyses excluding this study. Risk of bias in the randomized clinical trial was assessed according to the Jadad scale in the following domains: (1) random sequence generation; (2) allocation concealment; (3) blinding of participants and personnel; and (4) completed withdrawals and dropouts.

\section{Statistical analyses}

We pooled data and used Risk Ratios (RR) with 90 or 95\% confidential Intervals (CI) for dichotomous outcomes including (1) death; and (2) the composite endpoint (death, ICU admission, or mechanical ventilation). We 
Fig. 1 Flow diagram of choosing the appropriated articles.

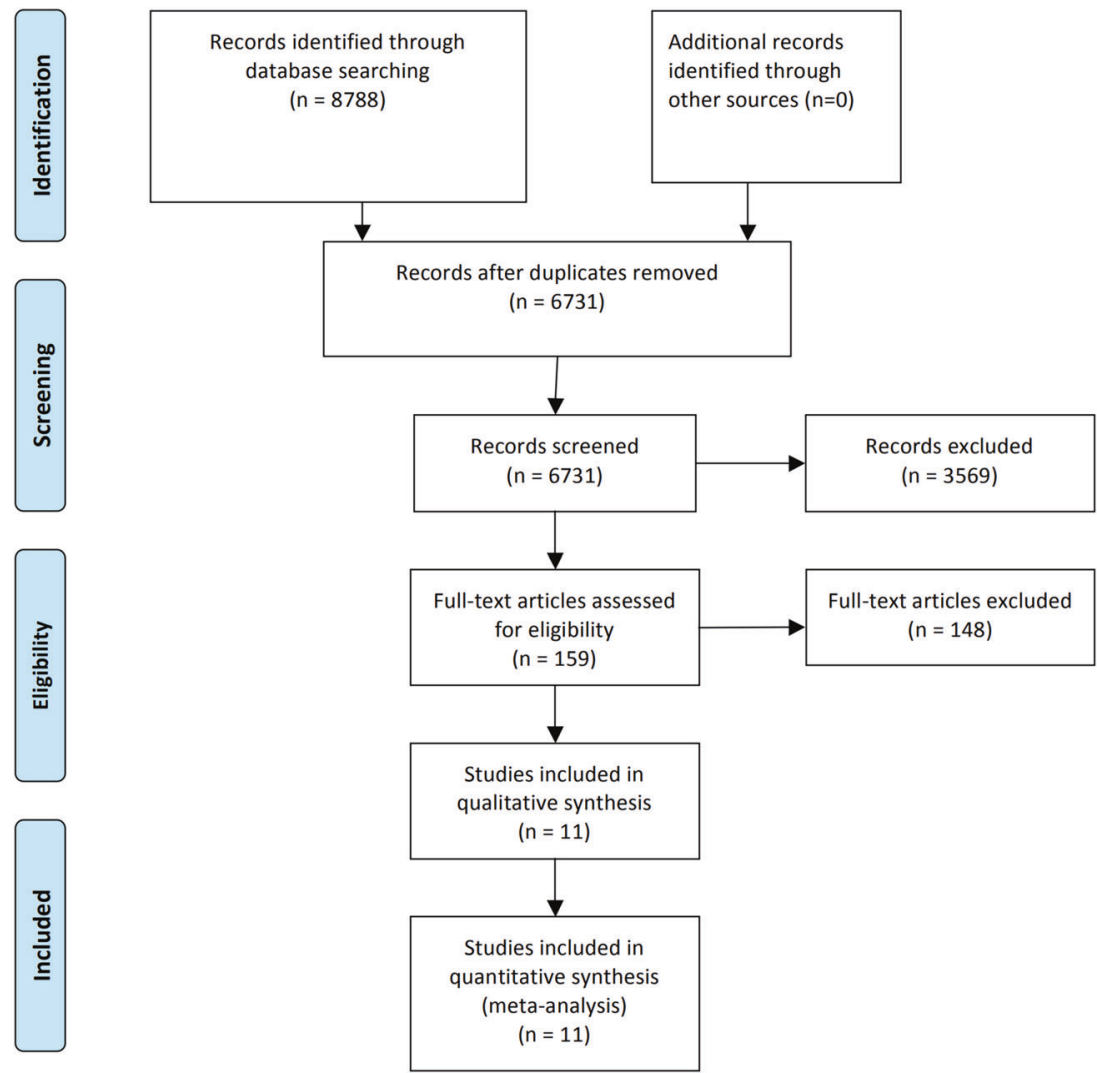

also used RR with $95 \%$ CI for dichotomous outcomes including (1) use of mechanical ventilation; and (2) corticosteroid application between severe-only and nonsevere subjects. If a study considered these as separate outcomes, we used death as the endpoint. Subgroup analyses divided by severity of disease were performed for these two types of outcomes. Definitions of severity of SARS used SARS guidelines of the Ministry of Public Health of China with a blood arterial oxygenation index $<300 \mathrm{~mm} \mathrm{Hg}$ [15]. Severity of COVID-19 was based on American Thoracic Society guidelines [19]. We used mean difference (MD) and 95\% CI for the continuous outcome including days to SARS-CoV-2 clearing, duration of hospitalization. Funnel plots were used to screen for potential publication bias. Statistical analyses used Review Manager 5.3 (The Cochrane Collaboration). Some studies [2, 11, 14, 15] identified subjects with nonsevere infection which we analyzed separately.

\section{Results}

The meta-analysis included 10 observational studies and 1 randomized clinical trials involving 5249 subjects. In three studies the publication language was Chinese [12-14] and six, English [2, 6, 8-11, 15, 16]. Six studies discussed SARS
[6, 11-15], four, COVID-19 [2, 8-10] and one, MERS [16]. Studies were published 2003-2020 and were conducted in China (Guangzhou [2, 15], Beijing [14], Shanxi [13], Hong Kong [6, 11], Wuhan [8-10]) and Saudi Arabia [16]. The Jadad scale of the randomized clinical trial was 4 and NOS scores of the observational studies was 5-9. One study was considered poor quality evidence because of brief follow-up (Table 1).

\section{Virus clearing}

To test the impact of corticosteroid use on virus clearing we included 2 studies [6, 16] of 81 subjects (SARS, $N=$ 16; MERS, $N=65)$. The data indicate corticosteroid use delayed virus clearing (MD 3.78 days, 95\% confidence interval [CI], 1.16, 6.41 days; $I^{2}=0 \%$; Fig. 2).

\section{Risk of death}

To test impact of corticosteroid use on risk of death, we included 8 studies [8-11, 13-16] involving 3909 subjects (COVID-19; $N=327$; SARS, $N=3273$; $\operatorname{MERS,~} N=$ 309). The data indicate corticosteroid did not significantly reduce risk of death $\left(\mathrm{RR}=1.07\right.$ [90\% CI, 0.81, 1.42; $I^{2}=$ $80 \%]$ ). To address heterogeneity we divided subjects into severe-only and other. RRs for severe-only and other 


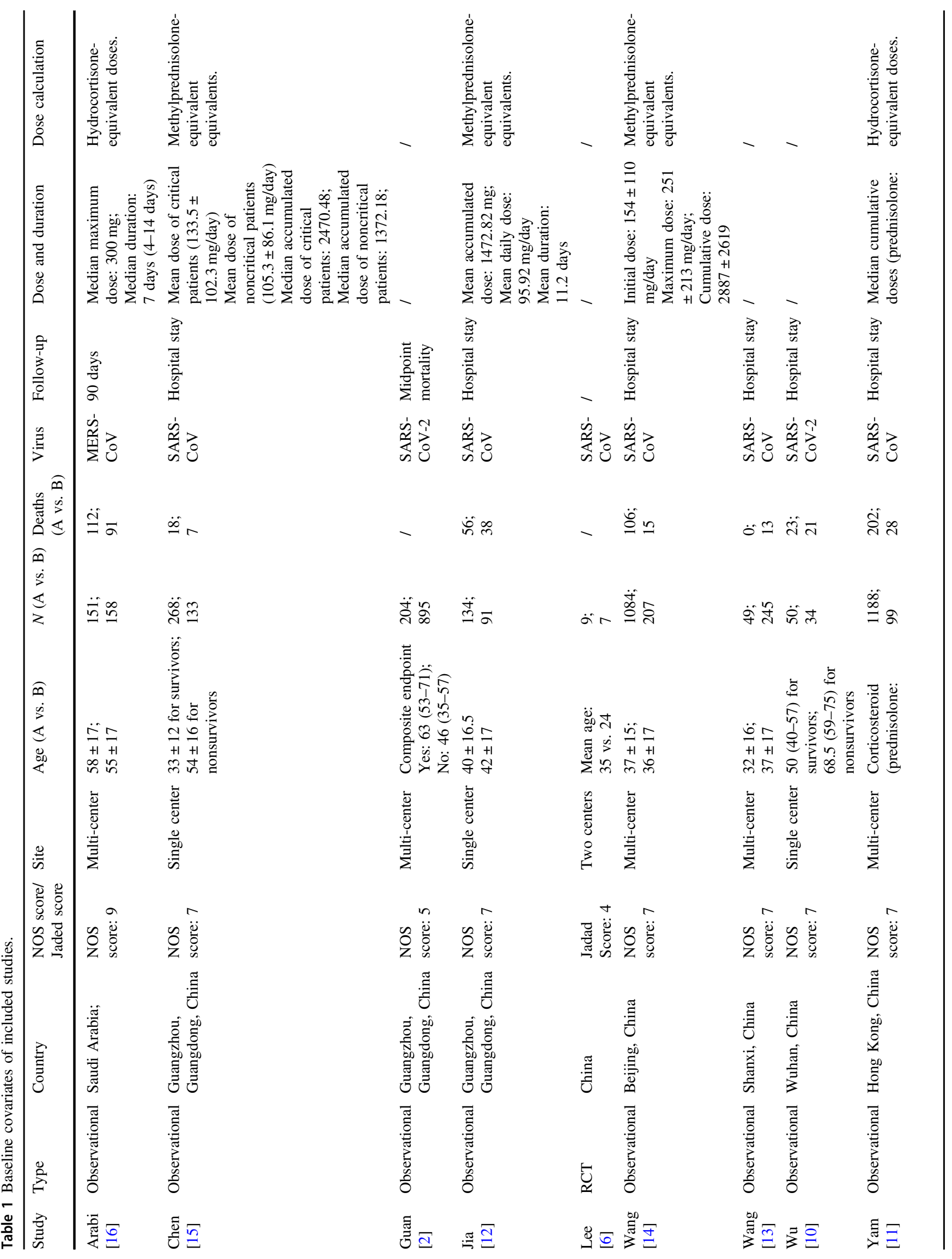




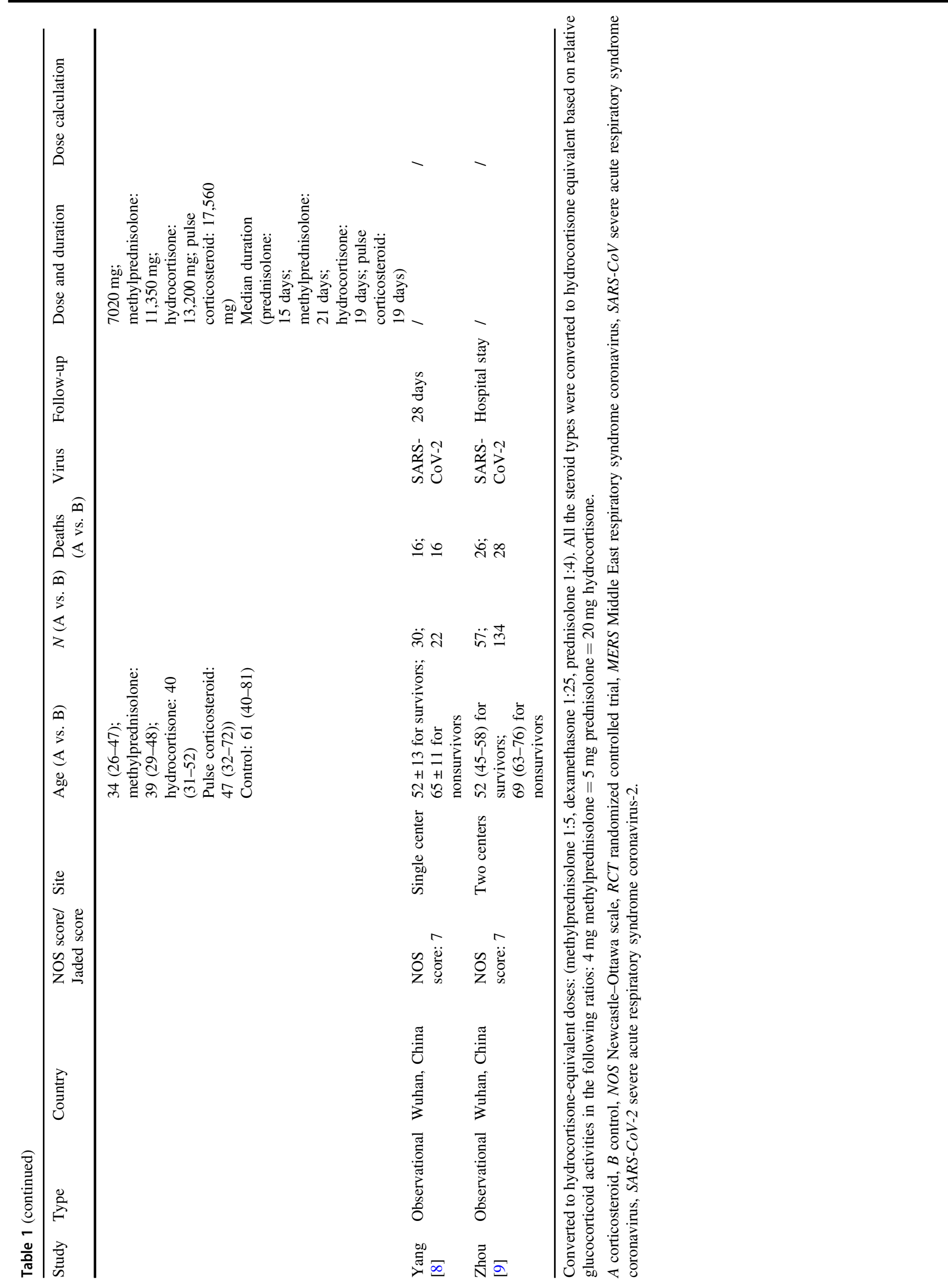




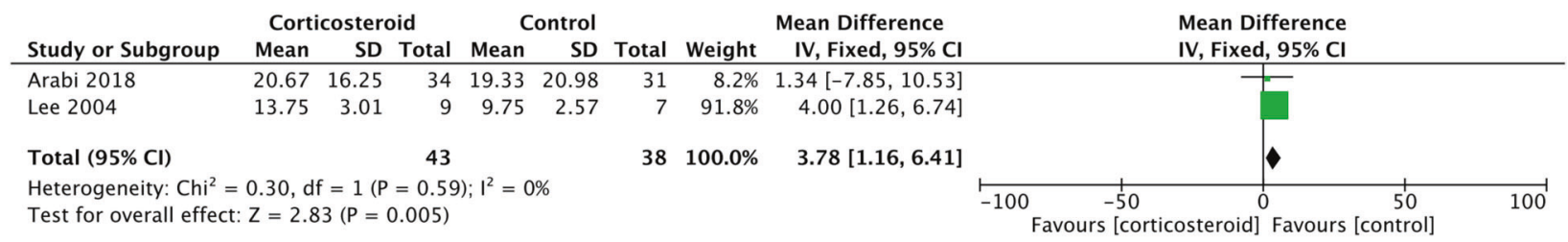

Fig. 2 The effect of corticosteroid on virus clearness. Comparison of virus clearness between corticosteroid and comparator.

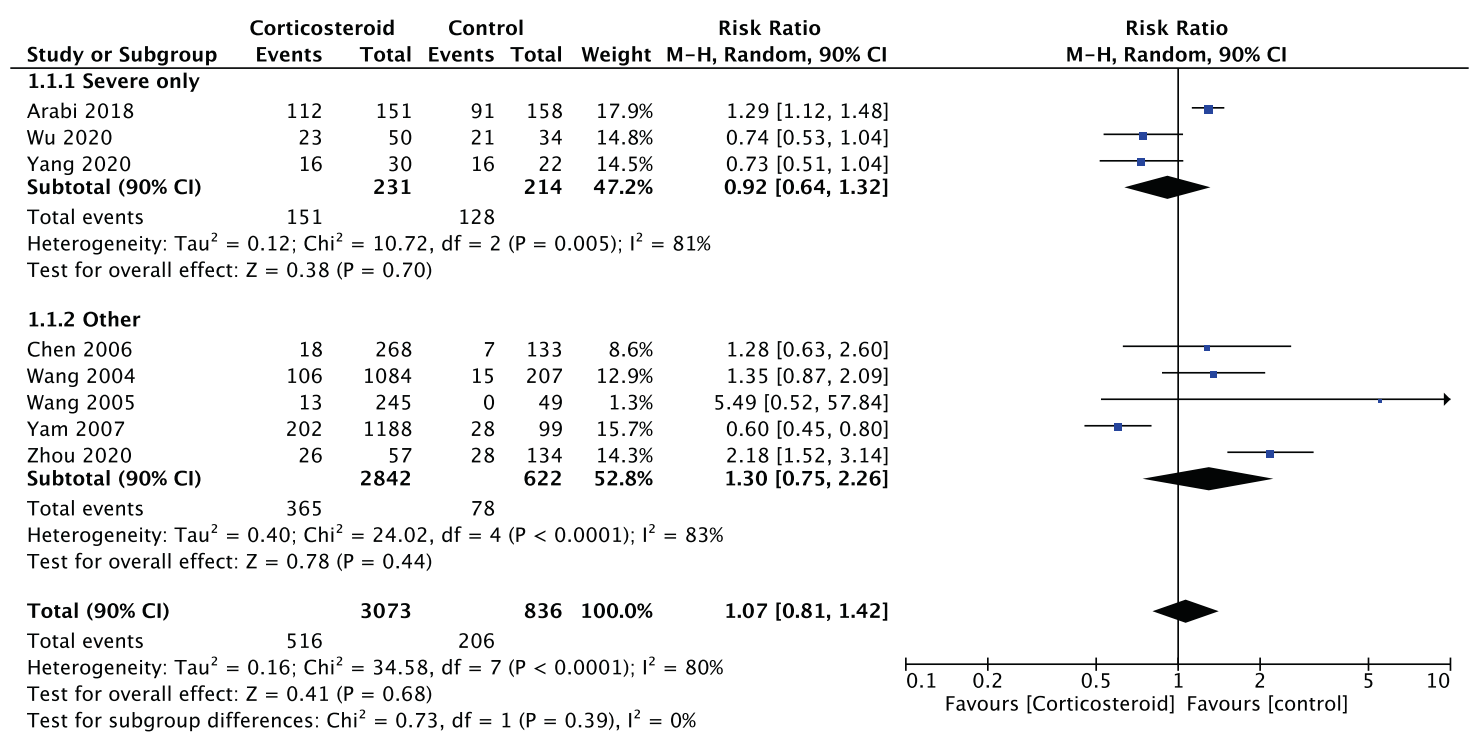

Fig. 3 The impact of corticosteroid on the mortality of studied subjects. Comparison of mortality between corticosteroid and comparator.

cohorts were $\mathrm{RR}=0.92\left(0.64,1.32 ; I^{2}=81 \%\right)$ and $\mathrm{RR}=$ $1.30\left(0.75,2.26 ; I^{2}=83 \%\right.$; Fig. 3$)$.

\section{Duration of hospitalization}

To test impact of corticosteroid use on duration of hospitalization we included 3 studies $[12,13,16]$ involving 828 subjects (SARS, $N=519$; MERS, $N=309$ ). The data indicate corticosteroid use increased duration of hospitalization with $\mathrm{MD}=9.66$ days (95\% CI, 5.15, 14.18 days); $I^{2}=85 \%$; Fig. 4 . We were unable to further analyze this endpoint by cohorts because of our study design.

\section{Use of mechanical ventilation}

To test impact of corticosteroid use on use mechanical ventilation we included 3 studies $[11,14,16]$ involving 2887 subjects (COVID-19, $N=2578$; MERS, $N=309$ ). The data indicate corticosteroid use is not associated with use of mechanical ventilation, $\mathrm{RR}=1.33\left(95 \% \mathrm{CI}, 0.73,2.42 ; I^{2}=\right.$ $70 \%$; Fig. 5). We were unable to determine whether subjects began receiving corticosteroids before or after use of mechanical ventilation because of our study design.

\section{Composite endpoint}

To test impact of corticosteroid use on the composite endpoint we included 9 studies [2, 8-11, 13-16] involving 5008 subjects (COVID-19, $N=1426$; SARS, $N=$ 3273; MERS, $N=309)$. The data indicate no improvement with corticosteroid use RR $=1.31\left(0.92,1.88 ; I^{2}=\right.$ $89 \%)$. RRs for the severe-only and other cohorts were $\mathrm{RR}=0.92\left(0.64,1.32 ; I^{2}=81 \%\right)$ and $\mathrm{RR}=1.73(0.90$, $3.31 ; I^{2}=91 \%$; Supplementary Fig. 1).

\section{Comparison of corticosteroid use between severe-only and nonsevere subjects}

To determine if corticosteroid use was more common in subjects with severe versus nonsevere infections we compared frequency of use in these cohorts. We included 4 studies $[2,11,14,15]$ involving 4078 subjects (SARS, $N=2979$; COVID-19, $N=1099$ ). The data indicate subjects with severe-only coronavirus infections were more likely receive corticosteroids with an $\mathrm{RR}=$ $1.48\left(95 \%\right.$ CI, 1.03, 2.13; $I^{2}=99 \%$; Supplementary Fig. 2). 


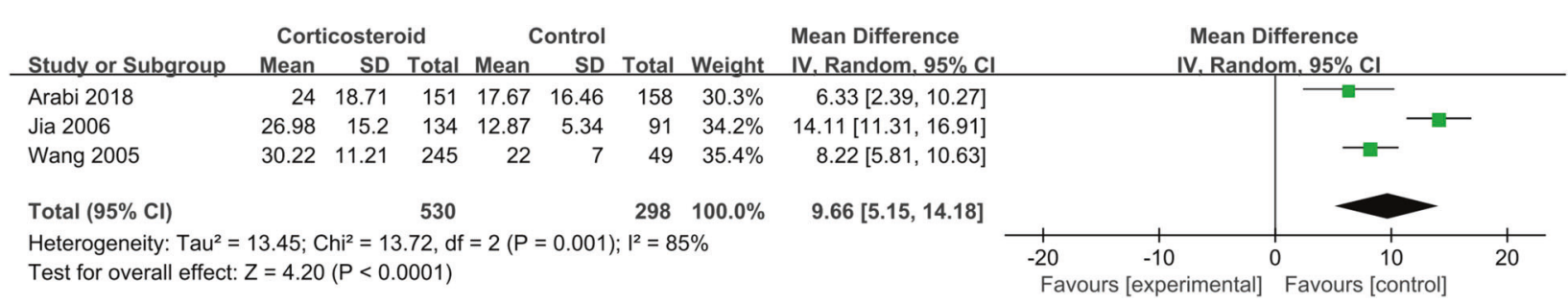

Fig. 4 The association of corticosteroid and hospital stay length. Comparison of hospital stay time between corticosteroid and comparator.

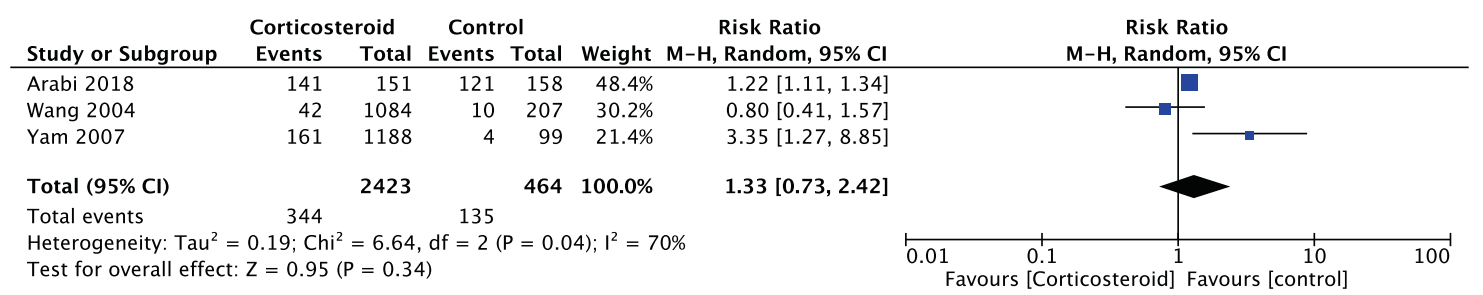

Fig. 5 The correlation between corticosteroid and invasive ventilation. Comparison of the incidence of invasive ventilation between corticosteroid and comparator.

\section{Publication bias}

We found potential publication bias in mortality studies in subjects receiving or not receiving corticosteroids with some studies falling outside the $95 \%$ CI of the funnel plat (Supplementary Fig. 3). There was publication bias in the studies included in the meta-analysis.

\section{Discussion}

SARS-CoV, MERS-CoV, and SARS-CoV-2 induce host immune responses which, when severe, damage the lungs and cause death $[3,20,21]$. Corticosteroids are frequently used to treatment persons with these coronavirus infections, especially when severe. Whether this strategy is safe and effective is controversial. Other approaches include counteracting high interleukin- 6 concentrations with drugs such as tocilizumab [22].

The results of our meta-analysis indicate giving corticosteroids in this setting delayed virus clearing of SARS-CoV and MERS-CoV. Whether this is so for SARS-CoV-2 is unknown. Our analysis of this issue included relatively few subjects so this conclusion should be viewed cautiously.

We determined corticosteroid use did not reduce deaths but was associated with increased hospitalization duration. There was no favorable impact on a composite endpoint of death, ICU admission, or mechanical ventilation. It was not possible to determine whether corticosteroid use preceded or followed use of mechanical ventilation. It is also important to emphasize associations and correlations do not imply cause-andeffect. Because most of the data come from observational databases and because corticosteroids are more likely to be given to persons with severe infections there is selection bias and our conclusions should be viewed cautiously. Also, because 6 of the 11 studies are from subjects with SARS-CoV and MERS-CoV our conclusions may not apply to SARS$\mathrm{CoV}-2$ infection. However, genetic homology of these viruses and similar, albeit not identical, clinical features of these infections make analyses of data from studies of SARS-CoV and MERS-CoV infections reasonable proxies.

A previous meta-analysis of corticosteroid use in persons with influenza (mostly H1N1) reported increased mortality [23]. Acute respiratory distress syndrome (ARDS) is the typical evolution of infectious pneumonia. It is unclear whether corticosteroids are effective in ARDS. Three cohort studies summarized in a meta-analysis reported corticosteroid use increased mortality in persons with influenzarelated ARDS [24]. In another study low- but not highdoses corticosteroids reduced mortality in persons with ARDS [25] suggesting dose may be an important co-variate in data analyses.

As indicated, the OR for death in subjects with severeonly coronavirus infection receiving corticosteroids is RR $=0.92\left(0.64,1.32 ; I^{2}=80 \%\right)$. How to interpret this central estimate and $90 \% \mathrm{CI}$ is controversial. Although the CI includes 1 and the $I^{2}$ is generally regarded as not significant (using an arbitrary cutoff significance level), the most conservative interpretation is that the results are inconclusive and consistent with as much as a $36 \%$ reduction or an almost 1.32-fold increase in risk of death. It is important to consider the absence of a statistically significant OR is not definitive evidence of risk or benefit. 
Safety and efficacy of corticosteroids in persons with SARS-CoV-2 infection COVID-19 can only be properly tested in a randomized clinical trial. However, such a trial is unlikely to be done. Our meta-analysis may help inform the decision whether to give corticosteroids.

\section{Conclusion}

Corticosteroid use in subjects with SARS-CoV-2, SARS$\mathrm{CoV}$, and MERS-CoV infections delayed virus clearing and did not convincingly improve survival, reduce hospitalization duration or ICU admission rate and/or use of mechanical ventilation. There were several adverse effects. Because of a preponderance of observational studies in the dataset and selection and publication biases our conclusions, especially regarding SARS-CoV-2, need confirmation in randomized clinical trials. In the interim we suggest caution using corticosteroids in persons with COVID-19. Our results also further consolidate the National Institute of Health (NIH) COVID-19 treatment guideline regarding corticosteroid interventions (https:// covid19treatmentguidelines.nih.gov/critical-care/pharma cologic-interventions/).

Funding YL was supported by the Program for Guangdong Introducing Innovative and Entrepreneurial Teams (2017ZT07S096) and the National Natural Science Foundation of China (Grant No. 81873428 and 81660682). RPG acknowledges support from the National Institute of Health Research (NIHR) Biomedical Research Centre funding scheme. Funding sources had input into the design, execution nor analyses of the data nor the decision to publish the results. Submission for publication was agreed by all authors all of whom had full access to of the data and take responsibility to submit the typescript for publication.

Author contributions YL and RPG designed study. HL and CXC searched databases and processed analysis. CXC, HL, YL, and RPG drafted the paper. YL, RPG, CXC, FH, JJW, and QYZ revised the final paper. YL and RPG are responsible for the paper.

\section{Compliance with ethical standards}

Conflict of interest The authors declare that they have no conflict of interest.

Publisher's note Springer Nature remains neutral with regard to jurisdictional claims in published maps and institutional affiliations.

\section{References}

1. Wang C, Horby PW, Hayden FG, Gao GF. A novel coronavirus outbreak of global health concern. Lancet. 2020;395:470-3.

2. Guan WJ, Ni ZY, Hu Y, Liang WH, Ou CQ, He JX, et al. Clinical characteristics of coronavirus disease 2019 in China. N Engl J Med. 2020;382:1708-20.
3. Huang C, Wang Y, Li X, Ren L, Zhao J, Hu Y, et al. Clinical features of patients infected with 2019 novel coronavirus in Wuhan, China. Lancet. 2020;395:497-506.

4. Chen N, Zhou M, Dong X, Qu J, Gong F, Han Y, et al. Epidemiological and clinical characteristics of 99 cases of 2019 novel coronavirus pneumonia in Wuhan, China: a descriptive study. Lancet. 2020;395:507-13.

5. Sung JJ, Wu A, Joynt GM, Yuen KY, Lee N, Chan PK, et al. Severe acute respiratory syndrome: report of treatment and outcome after a major outbreak. Thorax. 2004;59:414-20.

6. Lee N, Allen Chan KC, Hui DS, Ng EK, Wu A, Chiu RW, et al. Effects of early corticosteroid treatment on plasma SARSassociated Coronavirus RNA concentrations in adult patients. J Clin Virol. 2004;31:304-9.

7. Zhu N, Zhang D, Wang W, Li X, Yang B, Song J, et al. A novel coronavirus from patients with pneumonia in China, 2019. N Engl J Med. 2020;382:727-33.

8. Yang X, Yu Y, Xu J, Shu H, Xia J, Liu H, et al. Clinical course and outcomes of critically ill patients with SARS-CoV-2 pneumonia in Wuhan, China: a single-centered, retrospective, observational study. Lancet Respir Med. 2020. https://doi.org/10.1016/ S2213-2600(20)30079-5. Online ahead of print.

9. Zhou F, Yu T, Du R, Fan G, Liu Y, Liu Z, et al. Clinical course and risk factors for mortality of adult inpatients with COVID-19 in Wuhan, China: a retrospective cohort study. Lancet. 2020;395:1054-62.

10. Wu C, Chen X, Cai Y, Xia J, Zhou X, Xu S, et al. Risk factors associated with acute respiratory distress syndrome and death in patients with coronavirus disease 2019 pneumonia in Wuhan, China. JAMA Intern Med. 2020;e200994. https://doi.org/10.1001/ jamainternmed.2020.0994. Online ahead of print.

11. Yam LY, Lau AC, Lai FY, Shung E, Chan J, Wong V. Corticosteroid treatment of severe acute respiratory syndrome in Hong Kong. J Infect. 2007;54:28-39.

12. Jia W. Retrospectivestudy of the effect of glucocorticosteroids on the treatment of severe acute respiratory syndrome 2006 [D]. Sun Yat-sen University; 2006. http://www.wanfangdata.com.cn/deta ils/detail.do? type $=$ degree $\&$ id $=$ Y1014520.

13. Wang P, Li M, Shi Y, Wang S, Liu G. Evaluating the effects of different treatments on severe acute respiratory syndrome. Shanxi Med J. 2005;34:270-2.

14. Wang G, Li N, Wu Y, Xie G, Lin J, Huang C, et al. The Cox regression analysis on the use of corticosteroids in the treatment of SARS. Natl Med J China. 2004;84:1073-78.

15. Chen RC, Tang XP, Tan SY, Liang BL, Wan ZY, Fang JQ, et al. Treatment of severe acute respiratory syndrome with glucosteroids: the Guangzhou experience. Chest. 2006;129: 1441-52.

16. Arabi YM, Mandourah Y, Al-Hameed F, Sindi AA, Almekhlafi GA, Hussein MA, et al. Corticosteroid therapy for critically Ill patients with Middle East respiratory syndrome. Am J Respir Crit Care Med. 2018;197:757-67.

17. Taggart DP, D'Amico R, Altman DG. Effect of arterial revascularisation on survival: a systematic review of studies comparing bilateral and single internal mammary arteries. Lancet. 2001;358:870-5.

18. Fan X, Lin T, Xu K, Yin Z, Huang H, Dong W, et al. Laparoendoscopic single-site nephrectomy compared with conventional laparoscopic nephrectomy: a systematic review and meta-analysis of comparative studies. Eur Urol. 2012;62:601-12.

19. Metlay JP, Waterer GW, Long AC, Anzueto A, Brozek J, Crothers $\mathrm{K}$, et al. Diagnosis and treatment of adults with community-acquired pneumonia. an official clinical practice guideline of the American Thoracic Society and Infectious 
Diseases Society of America. Am J Respir Crit Care Med. 2019;200:e45-67.

20. Hui DSC, Zumla A. Severe acute respiratory syndrome: historical, epidemiologic, and clinical features. Infect Dis Clin North Am. 2019;33:869-89.

21. Almaghrabi RS, Omrani AS. Middle East respiratory syndrome coronavirus (MERS-CoV) infection. Br J Hosp Med. 2017;78:23-26.

22. Xu X, Han M, Li T, Sun W, Wang D, Fu B, et al. Effective treatment of severe COVID-19 patients with tocilizumab. Proc Natl Acad Sci U S A. 2020;202005615. https://doi.org/10.1073/ pnas.2005615117. Online ahead of print.
23. Rodrigo C, Leonardi-Bee J, Nguyen-Van-Tam J, Lim WS. Corticosteroids as adjunctive therapy in the treatment of influenza. Cochrane Database Syst Rev. 2016;3:Cd010406.

24. Ruan SY, Lin HH, Huang CT, Kuo PH, Wu HD, Yu CJ. Exploring the heterogeneity of effects of corticosteroids on acute respiratory distress syndrome: a systematic review and metaanalysis. Crit Care. 2014;18:R63.

25. Sun S, Liu D, Zhang H, Zhang X, Wan B. Effect of different doses and time-courses of corticosteroid treatment in patients with acute respiratory distress syndrome: a meta-analysis. Exp Ther Med. 2019;18:4637-44. 